\title{
Chaotic motion of particles in the accelerating and rotating black holes spacetime
}

\section{Songbai Chen, Mingzhi Wang and Jiliang Jing}

Institute of Physics and Department of Physics, Hunan Normal University, Changsha, Hunan 410081, People's Republic of China

Key Laboratory of Low Dimensional Quantum Structures and Quantum Control of Ministry of Education, Hunan Normal University, Changsha, Hunan 410081, People's Republic of China

Synergetic Innovation Center for Quantum Effects and Applications, Hunan Normal University, Changsha, Hunan 410081, People's Republic of China

E-mail: csb3752@hunnu.edu.cn, wmz9085@126.com, jljing@hunnu.edu.cn

ABSTRACT: We have investigated the motion of timelike particles along geodesic in the background of accelerating and rotating black hole spacetime. We confirm that chaos exists in the geodesic motion of the particles by Poincaré sections, the frequency spectrum and the power spectrum, the fast Lyapunov exponent indicator and the bifurcation diagram. Moreover, we probe the effects of the acceleration and rotation parameters on the chaotic behavior of a timelike geodesic particle in the black hole spacetime. Our results show that the acceleration brings richer physics for the geodesic motion of particles.

Keywords: Classical Theories of Gravity, Black Holes in String Theory

ArXiv EPrint: 1604.02785 


\section{Contents}

1 Introduction 1

2 Geodesic motion of a particle in the accelerating and rotating black hole spacetime

3 Chaotic phenomenon in the accelerating and rotating black hole spacetime

4 Summary

\section{Introduction}

Chaos is a kind of seeming random, chance or irregular motion, which appears only in the nonlinear and non-integrable dynamical systems. The most important feature of chaos is that it is highly sensitive to initial conditions [1-4]. The tiny errors in the chaotic motion can grow rapidly with time so that the motion is totally changed from what it would be in the absence of these errors, which means that it is very difficult to make a long-term prediction for chaotic motions in general. Thus, it is expected that chaotic systems possess many novel properties not shared by the usual dynamical systems, which triggers more attention to focus on the study of chaotic dynamics in various physical fields.

It is well known that the geodesic motion of particle in the generic Kerr-Newman black hole spacetime [5] is integrable and chaos does not emerge in this system. In order to study the chaotic motions of particles in general relativity, one must resort to some spacetimes with complicated geometries or introduce some extra interactions to ensure that the dynamical system describing motion of particle is non-integrable. Along this spirit, Cornish et al. [6, 7] investigated the chaotic trajectories of particle in multi-black hole spacetimes $[8,9]$ where the equations of motion of particle are not variable-separable. Moreover, the chaotic motions of particles have been studied in the perturbed Schwarzschild spacetime [10-13], or in the spacetime of a black hole immersed in magnetic field [14], or in the non-standard Kerr black hole spacetime described by Manko-Novikov metric [15-19]. The chaotic phenomenon was also found for the charged particles moving in a magnetic field interacting with gravitational waves [20]. More interestingly, Frolov and Larsen [21] showed that after introducing ring strings instead of point particles, one can find that the ring string dynamics is chaotic even in the asymptotically flat Schwarzschild black hole spacetime. Subsequently, the chaotic behavior of the ring string is also found in AdSSchwarzschild black hole [22] and AdS-Gauss-Bonnet black hole spacetimes [23].

In this paper, we will investigate the chaotic motion of particle in the accelerating and rotating black holes spacetime [24-26], which describes two uniformly separated Kerr-type 
black holes accelerating away from each other under the action of "strings" represented by conical singularities located along appropriate sections of the axis of symmetry. Comparing with the usual Kerr black hole, the accelerating and rotating black holes spacetime has different geometric structure because it possesses two rotation horizons and two acceleration horizons. This implies that both Hawking and Unruh radiation could be present in this background. Saifullah et al. [27, 28] studied the surface gravity, Hawking temperature and the area laws for accelerating and rotating black holes and explored the effects of the acceleration of black holes on the Hawking radiation of scalar particles in these black holes spacetime. We [29] investigated the collision of two massive geodesic particles in the accelerating and rotating black hole spacetime and probe the properties of the center-ofmass energy and high-velocity collision belts in the near horizon collision. Moreover, with this accelerating and rotating metric, Hawking and Ross [30] researched the possibility that a black hole pair can be created by the breaking of a cosmic string. Since the study of such a kind of black holes could provide physical insight into the high energy physics, the properties of the accelerating and rotating black holes have been investigated extensively in recent years [24-31]. The main purpose of this paper is to investigate the chaotic dynamics in the accelerating and rotating black holes spacetime.

The paper is organized as follows. In section 2, we briefly review the accelerating and rotating black hole spacetime and discuss the equations of geodesic motion for a timelike particle in this background. In section 3, we investigate the chaotic phenomenon in the accelerating and rotating black holes spacetime. We end the paper with a summary.

\section{Geodesic motion of a particle in the accelerating and rotating black hole spacetime}

The accelerating and rotating black hole spacetime describes the gravitational field by a pair of uniformly accelerating Kerr-type black holes, which is a special case of the Plebański and Demiański metric [32] covered a large family of electro-vacuum type- $D$ spacetimes including both the Kerr-Newman like solutions and the $C$-metric. In the Boyer-Lindquist coordinates, the metric of this accelerating and rotating black holes spacetime has a form [24-26]

$$
\begin{aligned}
d s^{2}= & -\left(\frac{\Delta-a^{2} P \sin ^{2} \theta}{\rho^{2} \Omega^{2}}\right) d t^{2}+\left(\frac{\rho^{2}}{\Delta \Omega^{2}}\right) d r^{2}+\left(\frac{\rho^{2}}{P \Omega^{2}}\right) d \theta^{2} \\
& +\left(\frac{\sin ^{2} \theta\left[P\left(r^{2}+a^{2}\right)^{2}-a^{2} \Delta \sin ^{2} \theta\right]}{\rho^{2} \Omega^{2}}\right) d \phi^{2} \\
& -\left(\frac{2 a \sin ^{2} \theta\left[P\left(r^{2}+a^{2}\right)-\Delta\right]}{\rho^{2} \Omega^{2}}\right) d t d \phi,
\end{aligned}
$$

with

$$
\begin{aligned}
\Omega & =1-\alpha r \cos \theta, \\
\rho^{2} & =r^{2}+a^{2} \cos ^{2} \theta, \\
P & =1-2 \alpha M \cos \theta+\alpha^{2} a^{2} \cos ^{2} \theta, \\
\Delta & =\left(r^{2}-2 M r+a^{2}\right)\left(1-\alpha^{2} r^{2}\right) .
\end{aligned}
$$


Here the parameters $M, \alpha$ and $a$ denote the mass, the acceleration and the angular momentum per unit mass of the black hole, respectively. The locations of black hole horizons are determined by equation $g^{r r}=0$ [24-26], i.e.,

$$
\frac{\Delta \Omega^{2}}{\rho^{2}}=0
$$

Solving above eqaution, one can obtain

$$
r_{H}=M+\sqrt{M^{2}-a^{2}}, \quad r_{C}=M-\sqrt{M^{2}-a^{2}}, \quad r_{A}=\frac{1}{\alpha}, \quad r_{\alpha}=\frac{1}{\alpha \cos \theta} .
$$

It is easy to find that the position of the event horizon $r=r_{H}$ and Cauchy horizon $r=r_{C}$ are same to those of the Kerr black hole. However, in this case, one can find that there also exist other two horizons at $r_{A}=\frac{1}{\alpha}$ and $r_{\alpha}=\frac{1}{\alpha \cos \theta}$, which are interpreted as the acceleration horizons in the context of the $C$-metric. Obviously, the presence of acceleration changes the geometry of spacetime. Unlike in the usual Kerr black hole spacetime, the physical region of the black hole is located in $r_{H}<r<r_{A}$ in which $\Delta>0$ is satisfied.

In the curve spacetime, the Lagrangian of a timelike particle moving along the geodesic is

$$
\mathcal{L}=\frac{1}{2} g_{\mu \nu} \dot{x}^{\mu} \dot{x}^{\nu}
$$

where the dots denote derivatives with respect to the proper time $\tau$. For the accelerating and rotating black hole spacetime (2.1), the Lagrangian takes the form

$$
\begin{aligned}
\mathcal{L}=\frac{1}{2}\{ & -\left(\frac{\Delta-a^{2} P \sin ^{2} \theta}{\rho^{2} \Omega^{2}}\right) \dot{t}^{2}+\frac{\rho^{2} \dot{r}^{2}}{\Delta \Omega^{2}}+\frac{\rho^{2} \dot{\theta}^{2}}{P \Omega^{2}}-\left(\frac{2 a \sin ^{2} \theta\left[P\left(r^{2}+a^{2}\right)-\Delta\right]}{\rho^{2} \Omega^{2}}\right) \dot{t} \dot{\varphi} \\
& \left.+\left(\frac{\sin ^{2} \theta\left[P\left(r^{2}+a^{2}\right)^{2}-a^{2} \Delta \sin ^{2} \theta\right]}{\rho^{2} \Omega^{2}}\right) \dot{\varphi}^{2}\right\} .
\end{aligned}
$$

Making use of the Euler-Lagrangian equation, we obtain the equations of motion of the timelike particles

$$
\begin{aligned}
& \dot{t}=\frac{g_{33} E+g_{03} L}{g_{03}^{2}-g_{00} g_{33}}=\frac{\Omega^{2}}{\Delta P \rho^{2}}\left\{\left[P\left(r^{2}+a^{2}\right)^{2}-a^{2} \Delta \sin ^{2} \theta\right] E-a\left[P\left(r^{2}+a^{2}\right)-\Delta\right] L\right\}, \\
& \dot{\varphi}=-\frac{g_{03} E+g_{00} L}{g_{03}^{2}-g_{00} g_{33}}=\frac{\Omega^{2}}{\Delta P \rho^{2} \sin ^{2} \theta}\left\{a \sin ^{2} \theta\left[P\left(r^{2}+a^{2}\right)-\Delta\right] E+a\left[\Delta-a^{2} P \sin ^{2} \theta\right] L\right\}, \\
& \ddot{r}=\frac{\Delta}{2 \rho^{2}}\left\{\left[\frac{2\left(\Delta-a^{2} P \sin ^{2} \theta\right)}{\rho^{2}}\left(\frac{\Omega_{, r}}{\Omega}+\frac{\rho_{, r}}{\rho}\right)-\frac{\Delta_{, r}-a^{2} P_{, r} \sin ^{2} \theta}{\rho^{2}}\right] \dot{t}^{2}+\frac{\rho^{2}}{\Delta}\left[\frac{\Delta_{, r}}{\Delta}+2\left(\frac{\Omega_{, r}}{\Omega}-\frac{\rho_{, r}}{\rho}\right)\right] \dot{r}^{2}\right. \\
& +\frac{\rho^{2}}{P}\left[2\left(\frac{\rho_{, r}}{\rho}-\frac{\Omega_{, r}}{\Omega}\right)-\frac{P_{, r}}{P}\right] \dot{\theta}^{2}+\frac{4 \rho^{2}}{\Delta}\left[\frac{\Omega_{, \theta}}{\Omega}-\frac{\rho_{, \theta}}{\rho}\right] \dot{r} \dot{\theta} \\
& -\frac{2 a \sin ^{2} \theta\left[P\left(r^{2}+a^{2}\right)-\Delta\right]}{\rho^{2}}\left[\frac{P_{, r}\left(r^{2}+a^{2}\right)+2 r P-\Delta_{, r}}{P\left(r^{2}+a^{2}\right)-\Delta}-2\left(\frac{\Omega_{, r}}{\Omega}+\frac{\rho_{, r}}{\rho}\right)\right] \dot{t} \dot{\varphi} \\
& +\frac{\sin ^{2} \theta\left[P\left(r^{2}+a^{2}\right)^{2}-a^{2} \Delta \sin ^{2} \theta\right]}{\rho^{2}} \\
& \left.\times\left[\frac{P_{, r}\left(r^{2}+a^{2}\right)^{2}+2 r\left(r^{2}+a^{2}\right) P-a^{2} \Delta_{, r} \sin ^{2} \theta}{P\left(r^{2}+a^{2}\right)^{2}-a^{2} \Delta \sin ^{2} \theta}-2\left(\frac{\Omega_{, r}}{\Omega}+\frac{\rho_{, r}}{\rho}\right)\right] \dot{\varphi}^{2}\right\},
\end{aligned}
$$




$$
\begin{aligned}
\ddot{\theta}=\frac{P}{2 \rho^{2}}\{[ & {\left[\frac{2\left(\Delta-a^{2} P \sin ^{2} \theta\right)}{\rho^{2}}\left(\frac{\Omega_{, \theta}}{\Omega}+\frac{\rho_{, \theta}}{\rho}\right)+\frac{a^{2} \sin \theta\left(P_{, \theta} \sin \theta+2 P \cos \theta\right)}{\rho^{2}}\right] \dot{t}^{2}+\frac{2 \rho^{2}}{\Delta}\left[\frac{\Omega_{, \theta}}{\Omega}-\frac{\rho_{, \theta}}{\rho}\right] \dot{r}^{2} } \\
& +\frac{\rho^{2}}{P}\left[\frac{P, \theta}{P}-2\left(\frac{\rho_{, \theta}}{\rho}-\frac{\Omega_{, \theta}}{\Omega}\right)\right] \dot{\theta}^{2}+\frac{\rho^{2}}{P}\left[2\left(\frac{\rho_{, r}}{\rho}-\frac{\Omega_{, r}}{\Omega}\right)-\frac{P_{, r}}{P}\right] \dot{r} \dot{\theta} \\
& -\frac{2 a \sin ^{2} \theta\left[P\left(r^{2}+a^{2}\right)-\Delta\right]}{\rho^{2}}\left[\frac{P_{, \theta}\left(r^{2}+a^{2}\right)}{P\left(r^{2}+a^{2}\right)-\Delta}-2\left(\frac{\Omega_{, \theta}}{\Omega}+\frac{\rho_{, \theta}}{\rho}-\frac{\cos \theta}{\sin \theta}\right)\right] \dot{t} \dot{\varphi} \\
+ & \frac{\sin ^{2} \theta\left[P\left(r^{2}+a^{2}\right)^{2}-a^{2} \Delta \sin ^{2} \theta\right]}{\rho^{2}} \\
& \left.\times\left[\frac{P_{, \theta}\left(r^{2}+a^{2}\right)^{2}-2 a^{2} \Delta \sin \theta \cos \theta}{P\left(r^{2}+a^{2}\right)^{2}-a^{2} \Delta \sin ^{2} \theta}-2\left(\frac{\Omega_{, \theta}}{\Omega}+\frac{\rho_{, \theta}}{\rho}-\frac{\cos \theta}{\sin \theta}\right)\right] \dot{\varphi}^{2}\right\},
\end{aligned}
$$

with the constraint condition

$H_{1}=P \dot{r}^{2}+\Delta \dot{\theta}^{2}-\frac{\Omega^{4}}{\rho^{4} \sin ^{2} \theta}\left\{P \sin ^{2} \theta\left[\left(r^{2}+a^{2}\right) E-a L\right]^{2}-\Delta\left[a \sin ^{2} \theta E-L\right]^{2}\right\}+\frac{\Delta P \Omega^{2}}{\rho^{2}}=0$.

Here $E$ and $L$ correspond to the energy and angular momentum of the timelike particle, respectively. It is obvious that in the case with the non-zero acceleration, i.e., $\alpha \neq 0$, the equations of motion (2.12), (2.13) and (2.14) can not be variable-separable and the corresponding dynamical system is non-integrable because it admits only two integrals of motion $E$ and $L$, which implies that the motion of the particle could be chaotic in the four-dimensional accelerating and rotating black hole spacetime (2.1).

\section{Chaotic phenomenon in the accelerating and rotating black hole space- time}

Chaos is a class of very complex motion without accurate definition at present. Usually, it can be understood as a kind of seeming random, chance or irregular movement appeared in a definiteness system with nonlinear interaction and it is very sensitive to initial value. The chaotic phenomenon in dynamical systems can be detected by many kinds of methods including the Poincaré surfaces of section, the Lyapunov characteristic exponents, the fast Lyapunov indicators (FLI), the power spectrum, the fractal basin boundaries, the bifurcation diagram, and so on.

In order to investigate the dynamical properties of a chaotic system with some coupled and complicated differential equations, we must resort to the numerical method with high precision because the motion of a particle in chaotic region is very sensitive to initial value and the numerical errors may produce a big difference between the numerical behavior and the real motion of particle. Here, we adopt to the corrected fifth-order Runge-Kutta method suggested in literatures [33-36], in which the velocities $(\dot{r}, \dot{\theta})$ are corrected in integration and the numerical deviation is pulled back in a least-squares shortest path. As in refs. [3336], the energy of the dynamical system (2.13) is subjected to the constraint $H_{1}=0$, which means that $H_{1}$ could be regarded as a conserved quantity. However, the numerical errors in the integral calculation could yield some deviations so that the numerical solution $(\dot{r}, \dot{\theta}, r, \theta)$ does not satisfy the constraint $H_{1}=0$. In order to solve this problem, one can introduce a dimensionless parameter $\xi$ to make a connection between the numerical velocities $(\dot{r}, \dot{\theta})$ 

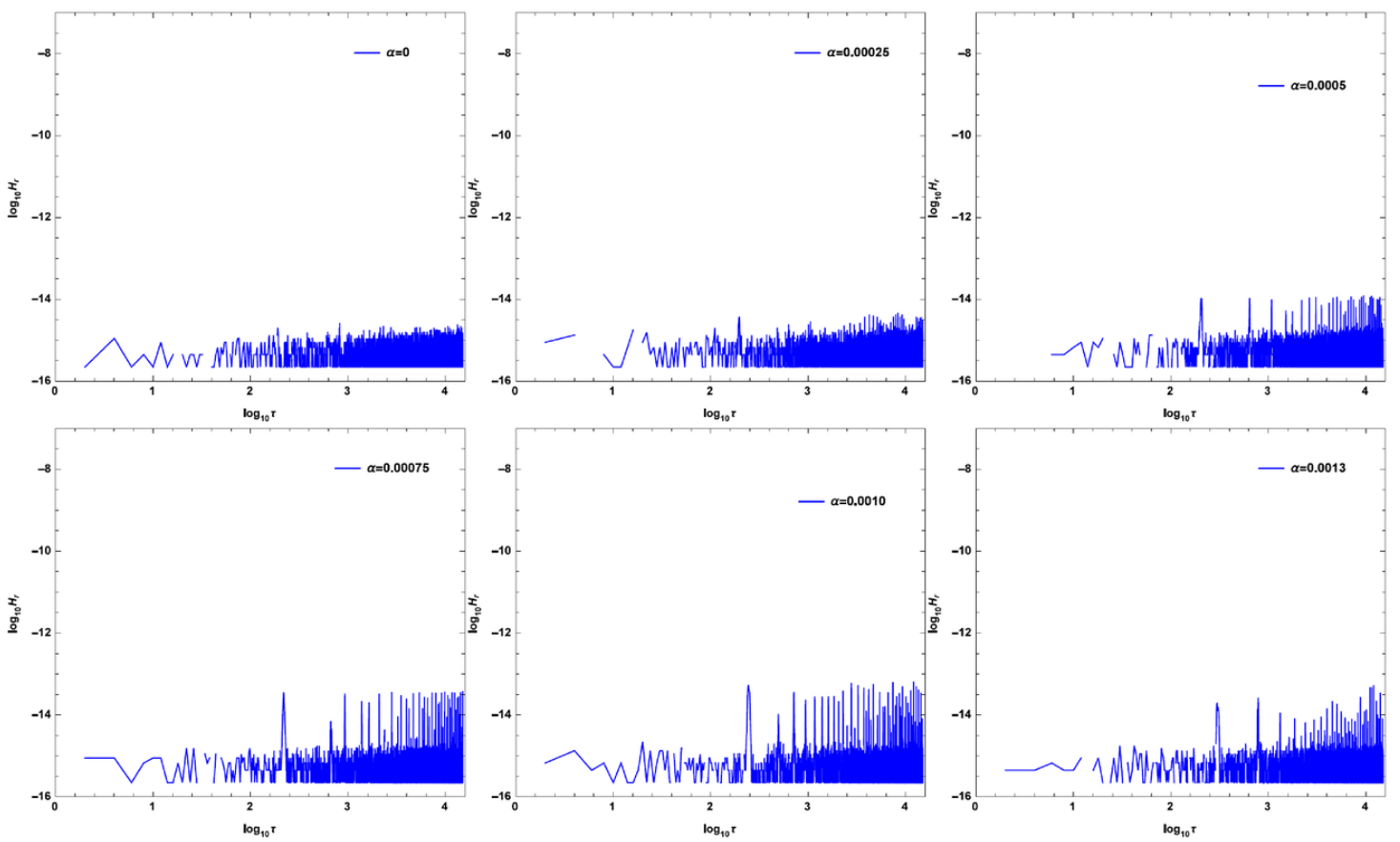

Figure 1. Errors of $H_{1}$ with time computed by the velocity correction method (RK5+Correction) in the accelerating and rotating black hole spacetime for different $\alpha$. Here, we set the parameters $E=0.95, L=3 M, a=0.6$, and the initial conditions $\left\{r(0)=8 ; \dot{r}(0)=0.1 ; \theta(0)=\frac{\pi}{2}\right\}$.

and the true value $\left(\dot{r}^{*}, \dot{\theta}^{*}\right)$ in the form of

$$
\dot{r}^{*}=\xi \dot{r}, \quad \dot{\theta}^{*}=\xi \dot{\theta} .
$$

The scale $\xi$ can be chosen such that the constraint (2.14) is always satisfied. Inserting eq. (3.1) into eq. (2.14), one can find that the scale factor of velocity correction $\xi$ in the accelerating and rotating black hole spacetime (2.1) is

$$
\xi=\sqrt{\frac{\frac{\Omega^{4}}{\rho^{4} \sin ^{2} \theta}\left\{P \sin ^{2} \theta\left[\left(r^{2}+a^{2}\right) E-a L\right]^{2}-\Delta\left[a \sin ^{2} \theta E-L\right]^{2}\right\}-\frac{\Delta P \Omega^{2}}{\rho^{2}}}{P \dot{r}^{2}+\Delta \dot{\theta}^{2}}} .
$$

In this way, the precision of the conserved quantity $H_{1}$ in the system of eqs. (2.10)-(2.14) at every integration step can hold perfectly. In figure 1 , we present the change of $H_{1}$ with time computed by the velocity correction method (RK5+Correction) in the accelerating and rotating black hole spacetime for different $\alpha$. Here, we set the parameters $E=0.95$, $L=3 M, a=0.6$, and the initial conditions $\left\{r(0)=8 ; \dot{r}(0)=0.1 ; \theta(0)=\frac{\pi}{2}\right\}$. From figure 1 , one can find that the value of $H_{1}$ is remained below $10^{-13}$ for different values of $\alpha$ and then the error is controlled greatly, which displays sufficiently that this correction method is very powerful so that it can avoid the pseudo chaos caused by numerical errors.

With help of the corrected fifth-order Runge-Kutta method, we present some solutions for $r(\tau)$ for different values of $\alpha$ in figure 2, which are obtained under the rotation parameter 

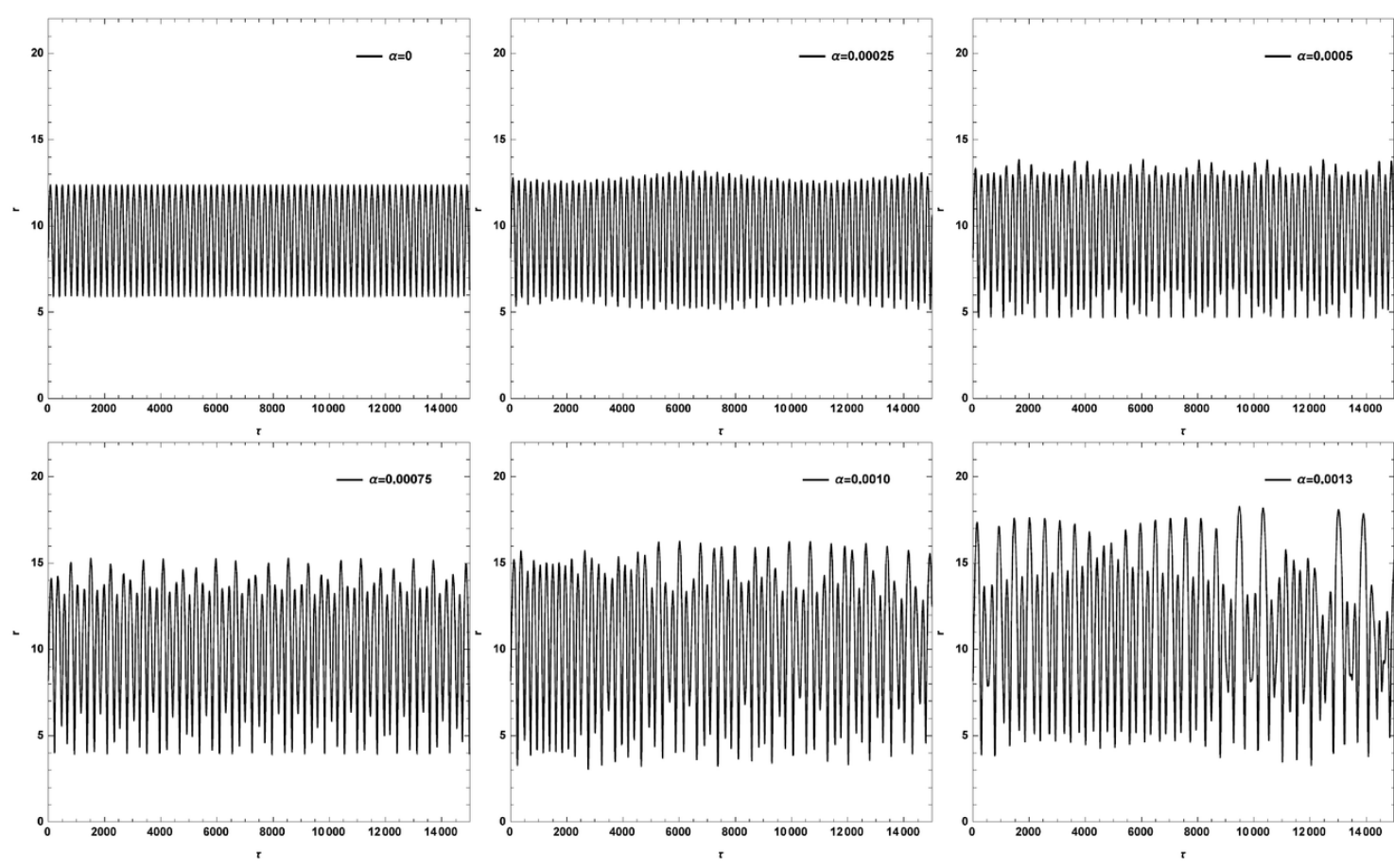

Figure 2. The change of $r(\tau)$ with $\tau$ for different $\alpha$ in the accelerating and rotating black hole spacetime with the parameters $E=0.95, L=3 M$, and $a=0.6$. The initial conditions are set by $\left\{r(0)=8 ; \dot{r}(0)=0.1 ; \theta(0)=\frac{\pi}{2}\right\}$.

$a=0.6$ and the set of initial conditions $\left\{r(0)=8 ; \dot{r}(0)=0.1 ; \theta(0)=\frac{\pi}{2}\right\}$. It is shown that the solution with $\alpha=0$ presented in figure 2 is a periodic solution and is not chaotic, which is explained by a fact that in the case $\alpha=0$ the metric (2.1) reduces to the usual Kerr black hole spacetime in which the timelike geodesic equations are variable-separable and the chaos does not emerge in such an integrable dynamical system. However, for the cases with $\alpha \neq 0$, we find that the amplitude and frequency components increase with $\alpha$. Especially, as $\alpha \geq 0.001$, it is difficult to describe the amplitudes and frequencies of this oscillation with any definite pattern, which implies that the motion could be chaotic. Thus, the presence of acceleration make the motion of particle more complicated. We also plot the phase curve in the $(r, \dot{r})$ plane of the phase space for this trajectory in figure 3 . It is shown that the phase path in the case with $\alpha=0$ is simple and is only a closed curve, which means that the corresponding solution is periodic and the particle moves along the stable periodic orbit around the black hole. However, with the increase of $\alpha$, the phase path becomes more complex and the region fulled by the path is enlarged, which means that the degree of disorder and non-integrability of the motion of particle increases with the acceleration parameter $\alpha$ in a sense for the signals plotted in figure 2. For the cases with $\alpha \geq 0.001$, one can find that the complex path fulls densely a given region in the phase plane. It is a typical feature of chaotic behaviors in the definiteness systems and then the chaotic motion occurs in these cases. For the case $\alpha>0.0016$, we obtain only a kind of unstable escaped solutions for the chosen initial conditions, which describe that 

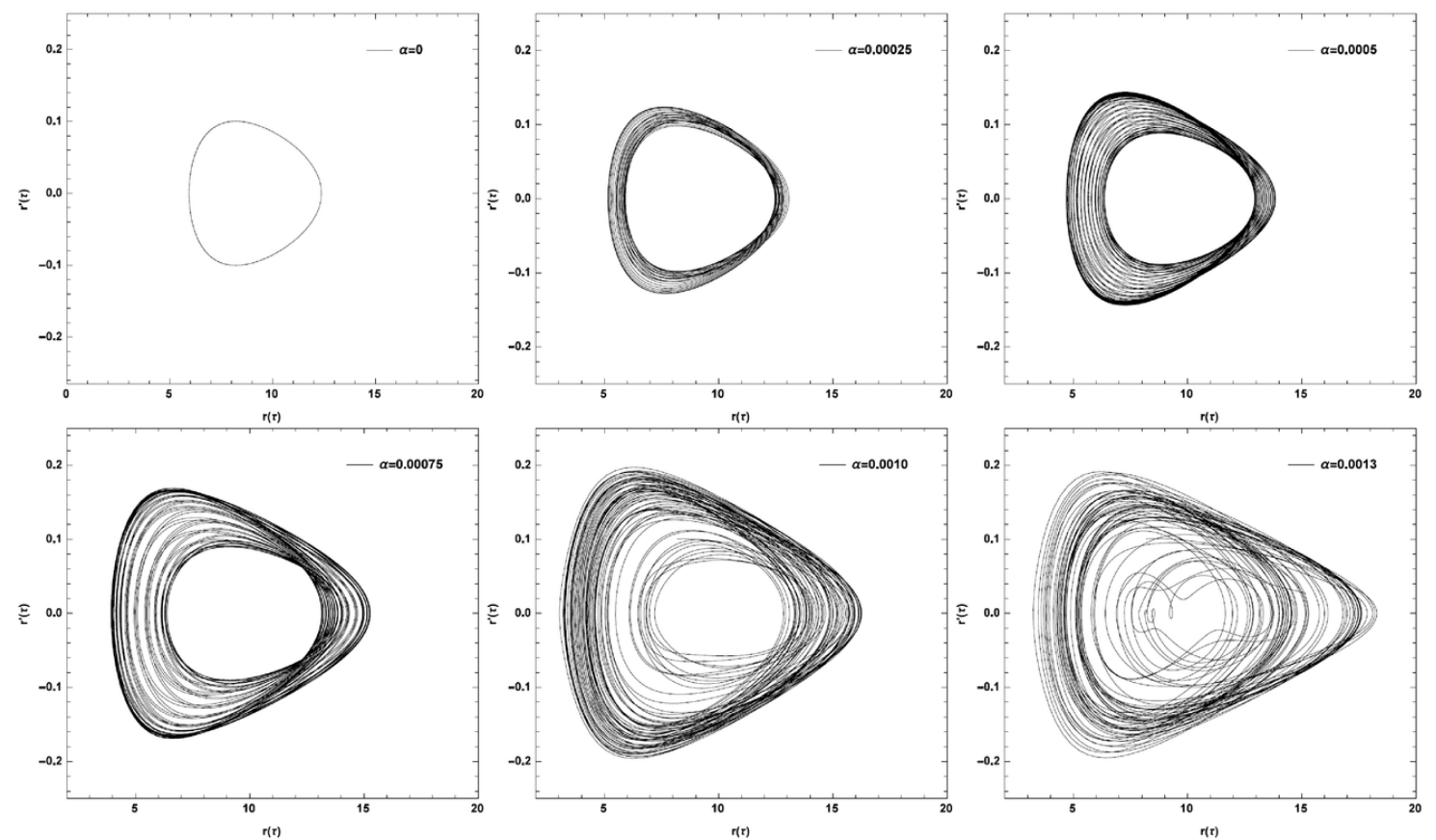

Figure 3. Phase curves corresponding to the solutions plotted in figure 2, projected into the $(r, \dot{r})$ plane.

the particle falls into the acceleration horizon or the event horizon after undergoing several chaotic oscillations around black hole. This could be explained by a fact that for the larger $\alpha$ the acceleration horizon $r_{A}$ is closer to the event horizon $r_{H}$ so that the particle is outoff-balance in the physical region around the black hole $\left(r_{H}<r<r_{A}\right)$. Thus, we will focus on the cases with $0 \leq \alpha<0.0016$ in this paper.

Let us now to analyze the frequency components of the signals with different values of $\alpha$ plotted in figure 2. Through the fast Fourier transformation, we present the spectrogram for the above signals in figure 4. The height of peaks are related to the amplitude of the corresponding frequency in a Fourier decomposition [1, 2]. In figure 4, one can find that there exists a high peak at the frequency $f=0 \mathrm{~Hz}$ in each panel, whose amplitude describes the average orbital radius of the particle moving around the black hole. For the signal with $\alpha=0$ in figure 2 , we also find that the peaks appear at the points with the frequency $f=f_{0}, 2 f_{0}, 3 f_{0}$ and $4 f_{0}$, respectively. Here $f_{0}$ is the lowest frequency and its numerical value is 0.004772 . It is a discrete spectrum, which means that in the case $\alpha=0$ the motion of particle is multiple-periodic motion rather than a simple single periodic motion for chosen parameters and initial conditions. However, comparing with the amplitude of fundamental frequency part, the amplitudes of the overtone frequency parts are very small, which yields that the phase path looks like a close curve in the $r-\dot{r}$ plane as in the case of single periodic motion. Figure 4 tells us that with the increase of $\alpha$, the frequency components increase and spectral lines become more dense. As $\alpha=0.001$, we find that the distinct continuous spectrum appears, which means that the motion of particle is chaotic in this case. As $\alpha=0.0013$, the width of continuous spectrum increases and the strength 

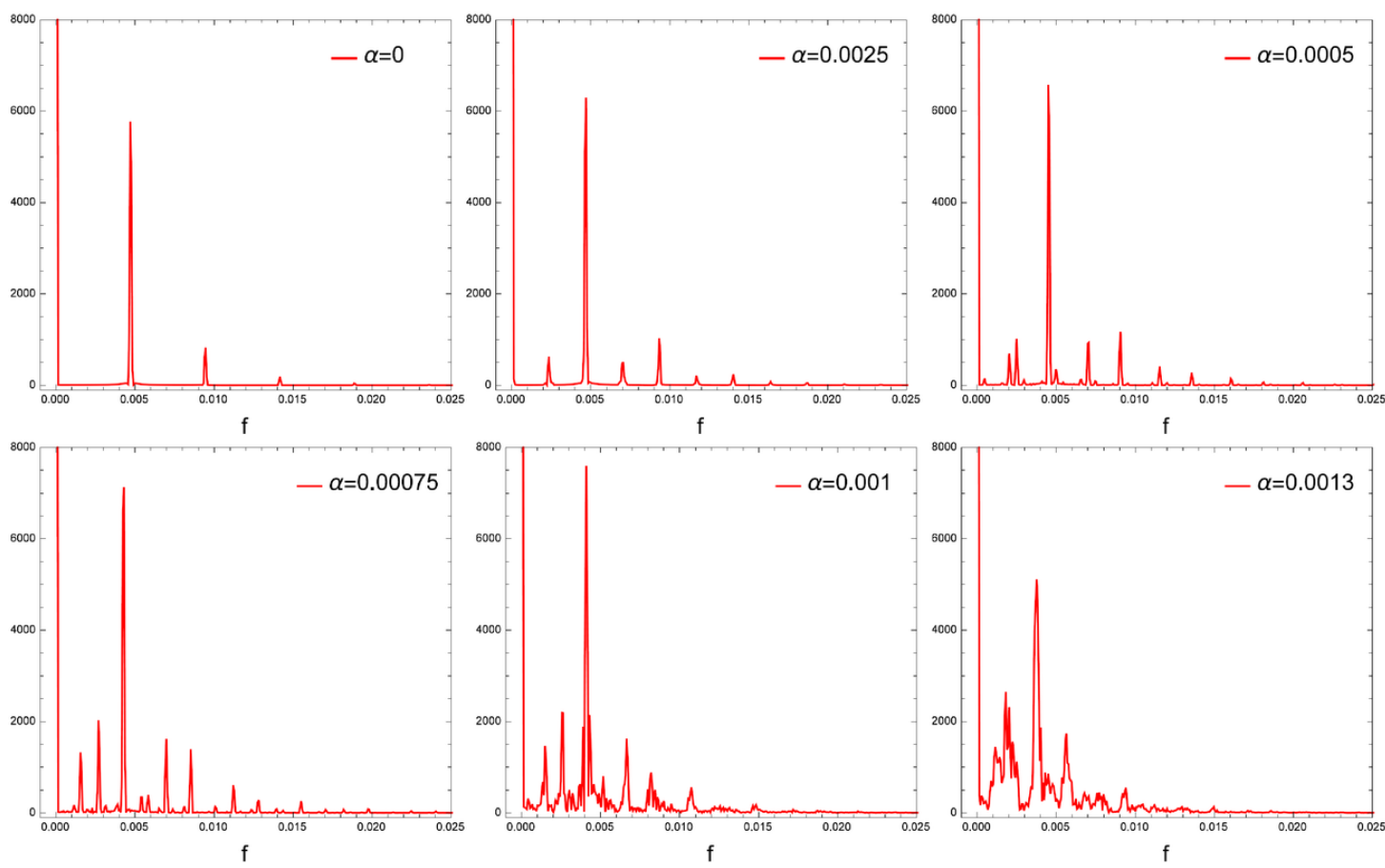

Figure 4. The frequency spectrum for the signals with different $\alpha$ plotted in figure 2 .

of chaotic motion enhances. The similar results are also obtained by analyzing the power spectrum shown in figure 5 .

The Lyapunov indicator is a kind of useful tools to identify whether the motion of particles are chaotic or not $[1,37-39]$. It is well known that the behavior of dynamical system is chaotic if the largest Lyapunov exponent is positive and is non-chaotic if the largest Lyapunov exponent is negative. In general, in order to obtain the Lyapunov exponent, one has to spend a long computational time even for a chaotic orbit since it is an infinite-time quantity. However, it has been shown that the relevant information on distinguishing between regular and chaotic trajectories could be obtained by integrating the equations of motion for a short time [40]. FLI is such a kind of faster and more sensitive indicators to reveal chaos orbits of particles. Froeschlé and Lega [41] describe the FLI as $\operatorname{FLI}(\tau)=\ln |Y(\tau)|$, where $Y(\tau)$ is a tangential vector of the flow at time $\tau$ as the particle moves along the trajectory. For a continuous flow along the trajectory including the periodic orbits, there exists a differential rotation which induces that the angle between the vectors of an initial orthonormal basis decreases sharply [40-42]. Due to the conservation of volume for the continuous flow in the phase space, the differential rotation leads to a rapid increase of the tangential vector $Y(\tau)$ of the flow [40-42], which means that FLI blows up for late times. The investigation [40-42] show that $\operatorname{FLI}(\tau)$ grows with exponential rate for chaotic motion, even for weak chaotic motion, and grows algebraically with time for the regular resonant orbit and for the periodic one. Since the deviation vector $\Delta X$ between two nearby trajectories can approximate well the tangent vector, FLI can also be simplified as $\operatorname{FLI}(\tau)=\ln \frac{|\Delta X(\tau)|}{|\Delta X(0)|}$. This is so-called the two-particle method or two-nearby-trajectories 

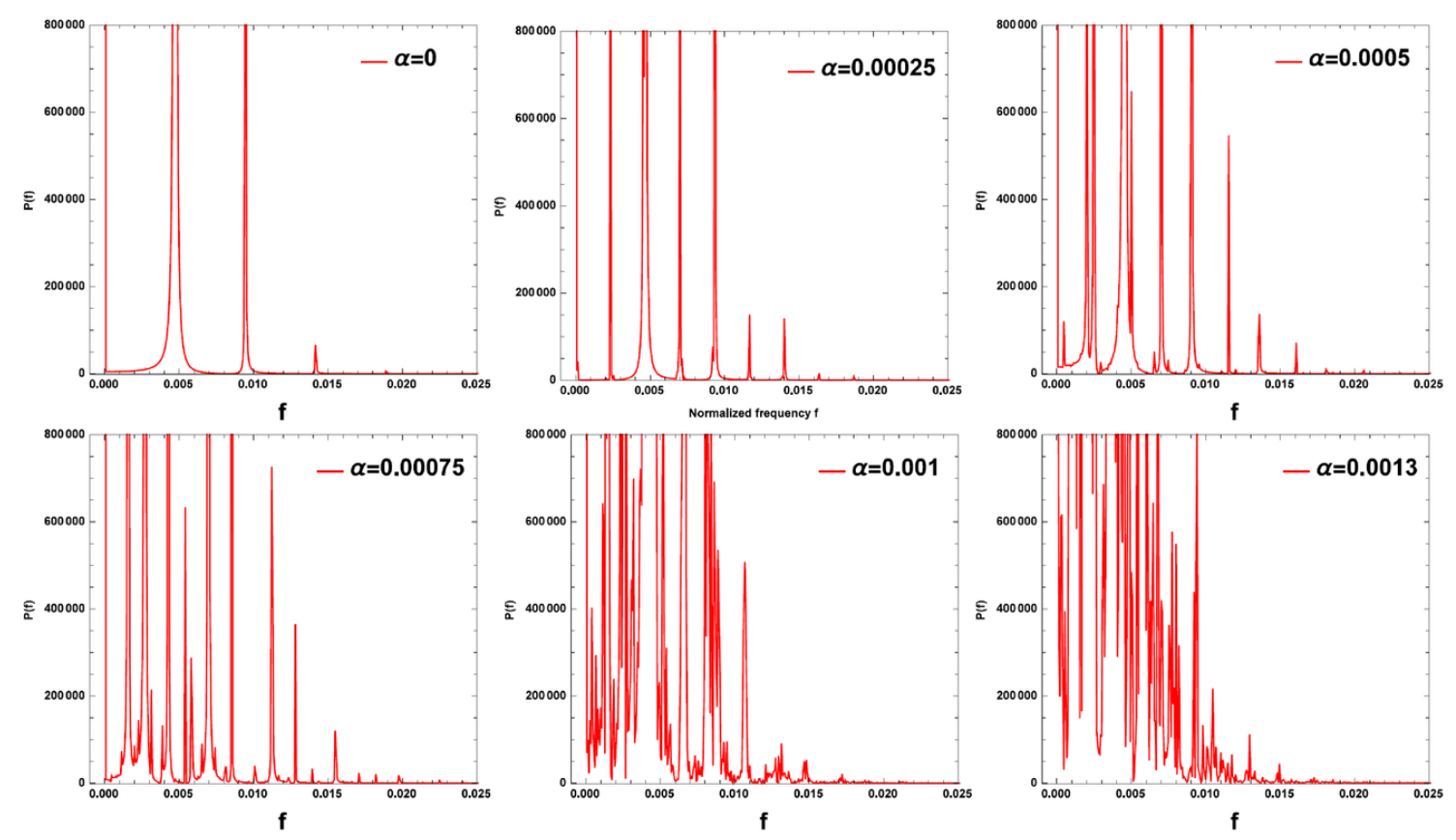

Figure 5. The power spectrum for the signals with different $\alpha$ plotted in figure 2 .

method $[43,44]$. It is a less rigorous but still useful technique. The version of FLI with two-nearby-trajectories in general relativity is described as [23, 45-47]

$$
\operatorname{FLI}(\tau)=-k\left[1+\log _{10} d(0)\right]+\log _{10}\left|\frac{d(\tau)}{d(0)}\right|
$$

where $d(\tau)=\sqrt{\left|g_{\mu \nu} \Delta x^{\mu} \Delta x^{\nu}\right|}, \Delta x^{\mu}$ is the deviation vector between two nearby trajectories at proper time $\tau$. The parameter $k$ is the sequential number of renormalization which is used to avoid that the two orbits expand too fast. In figure 6 , we present $\operatorname{FLI}(\tau)$ for the signals plotted in figure 2. It is shown that with increase of time $\tau, \operatorname{FLI}(\tau)$ grows with exponential rate for the signals with $\alpha=0.001$ and $\alpha=0.0013$, but with polynomial rate in the cases with $\alpha<0.001$. This confirms further that in figure 2 the orbital in with $\alpha=0.001$ and $\alpha=0.0013$ are chaotic and the orbital with $\alpha<0.001$ is ordered.

Poincaré section is another useful tool for analyzing dynamical systems. It is defined as an intersection of trajectory of a continuous dynamical system with a given hypersurface which is transversal to the trajectory in the phase space. In general, the solutions of the continuous dynamical system with different initial conditions can be classified as three kinds by the intersection points in a Poincaré section. One of them are periodic solutions, which are corresponds to a finite number of points in the Poincaré section. The quasiperiodic solutions correspond to a series of close curves and the chaos solutions correspond to strange patterns of dispersed points with complex boundaries.

In figure 7 , Poincaré sections with $\theta=\frac{\pi}{2}$ on the plane $(r, \dot{r})$ for different acceleration parameters $\alpha$ are plotted for the motion of a timelike particle in the accelerating and rotating black hole spacetime with the fixed parameters $a=0.6, E=0.95, L=3 M$ and 

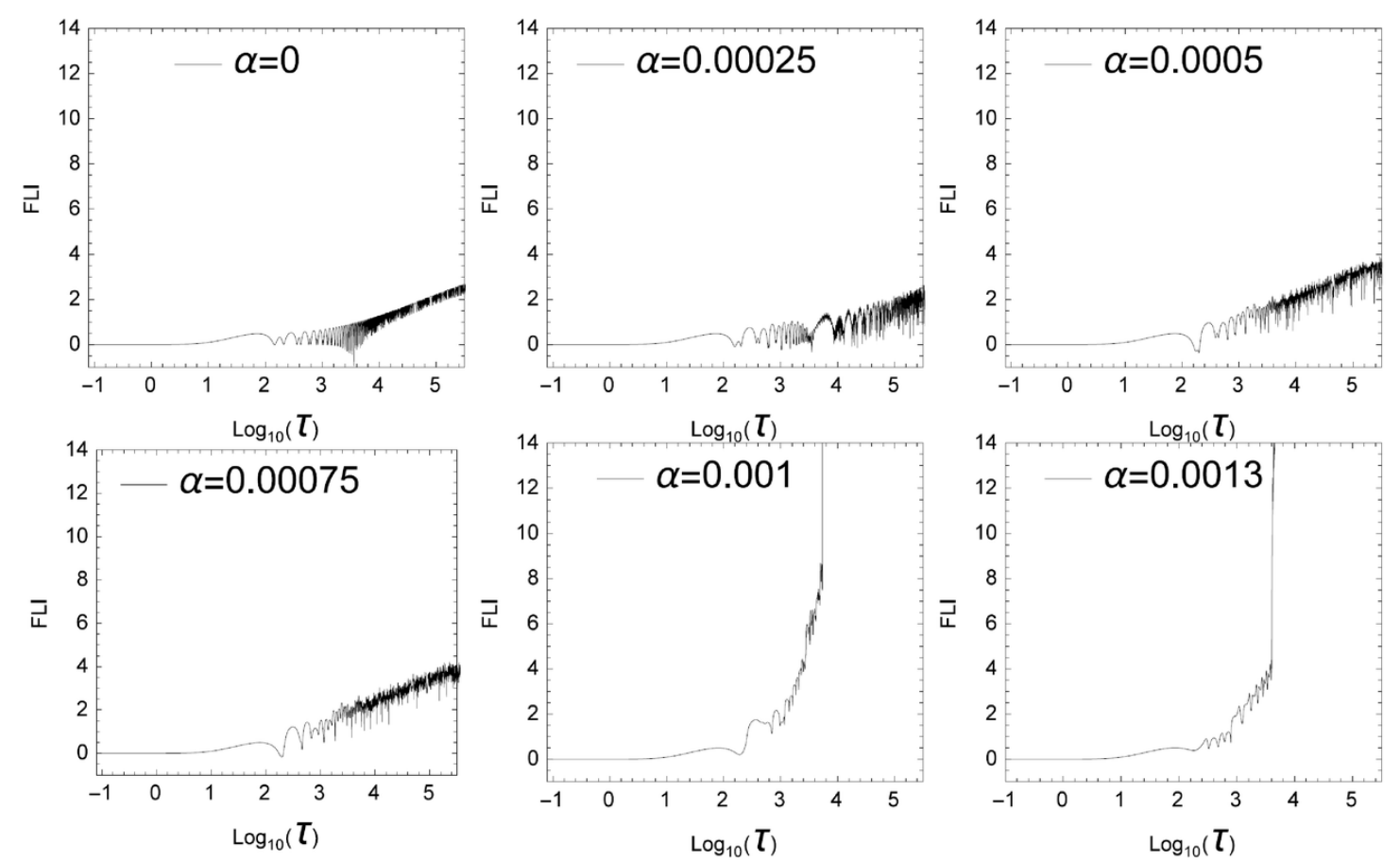

Figure 6. The Fast Lyapunov indicators with two nearby trajectories for the solutions with different $\alpha$ plotted in figure 2 .

the initial conditions $\left\{r(0)=8 ; \dot{r}(0)=0.1 ; \theta(0)=\frac{\pi}{2}\right\}$. We find that for $\alpha \in[0,0.009]$ the phase path is a quasi-periodic Kolmogorov-Arnold-Moser (KAM) tori and the behavior of this system is non-chaotic. Especially, there are more complicated KAM tori trajectories for $\alpha \in[0.000182,0.0004]$. It is composed of three secondary KAM tori belonging to the same trajectories where the successive points jump from one loop to the next. These little loops are called a chain of islands. With the acceleration parameter $\alpha$ increasing, the chain of islands are joined together and become a big KAM tori. This shows that trajectory is regular and integrable in this case. However, when $\alpha$ is larger than 0.0009 , KAM tori is destroyed and the corresponding trajectory is non-integrable, which indicates that the behavior of this system is chaotic. Especially, for $\alpha=0.001 \sim 0.0013$, the tori is completely destroyed and the pattern is composed of discrete points, which means that the chaotic behavior becomes stronger with the acceleration $\alpha$. From figure 7, we also note that there exist a few discrete points in the Poincaré section in the case with $\alpha=0.0015$, which is caused by a fact that the particle falls finally into the acceleration horizon of the black hole after undergoing several chaotic oscillations around black hole in this case. Thus, it is different essentially from those in the case of usual multiple-periodic motion. It is well known that the behavior of non-linear dynamical system depends on the choice of the initial conditions. In figure 8, we adopt another initial conditions $\{r(0)=12$; $\left.\dot{r}(0)=0.1 ; \theta(0)=\frac{\pi}{2}\right\}$ and investigate the dependence of Poincaré sections (with $\theta=\frac{\pi}{2}$ ) on the acceleration parameters $\alpha$. It is shown that with the increase of value of $\alpha$, KAM tori in the phase plane is destroyed gradually to dispersed points at first, and then it 

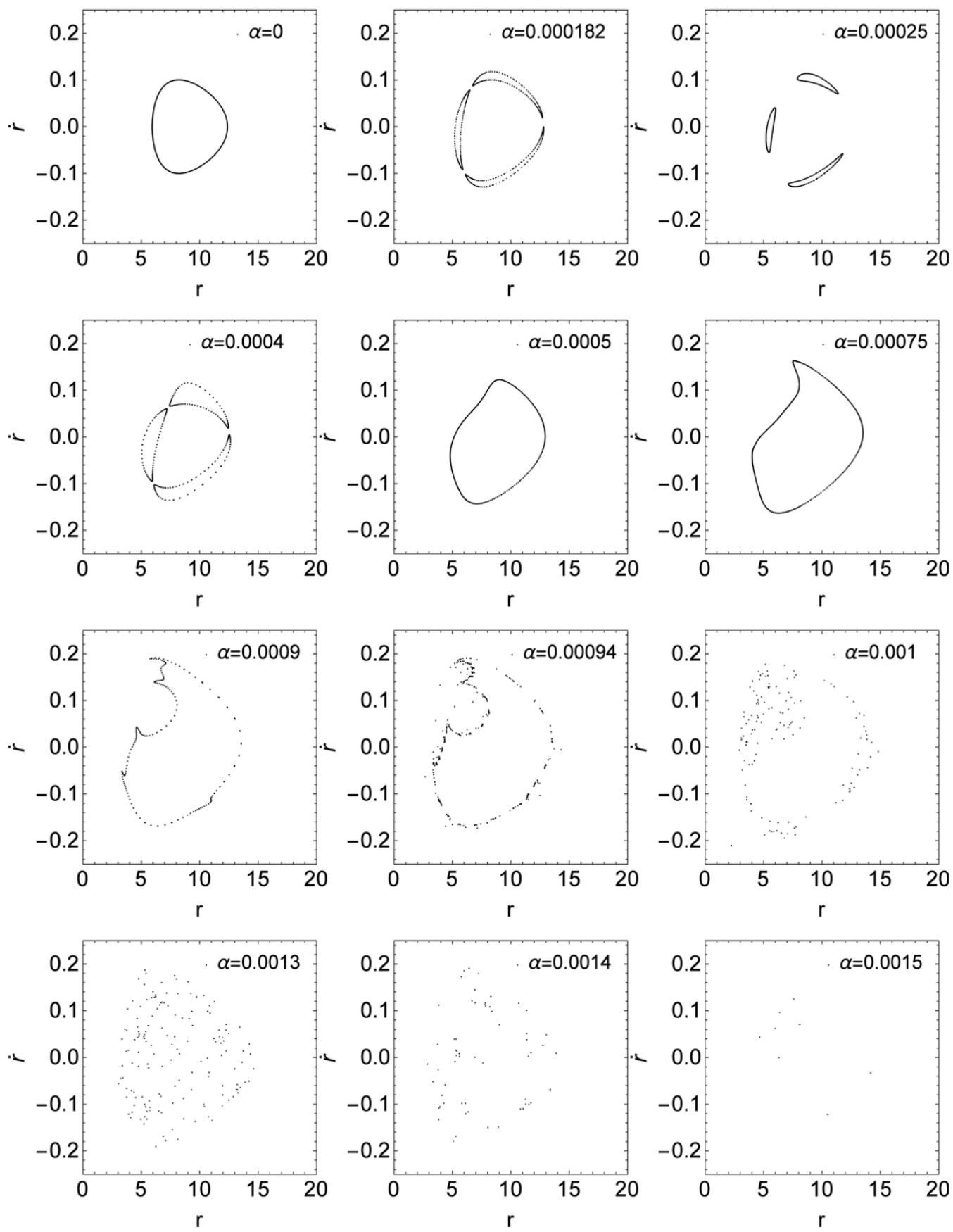

Figure 7. The change of Poincaré section $\left(\theta=\frac{\pi}{2}\right.$ on the plane $\left.(r, \dot{r})\right)$ with acceleration parameter $\alpha$ for the motion of the timelike particle in the accelerating and rotating black hole spacetime with the fixed parameters $a=0.6, E=0.95, L=3 M$ and the initial conditions $\{r(0)=8 ; \dot{r}(0)=0.1$; $\left.\theta(0)=\frac{\pi}{2}\right\}$.

is recovered slowly to a close curve. With further increasing of $\alpha$, the close KAM tori is completely destroyed again. This means that the behavior of the system undergoes a process from regular to chaotic then to regular, and finally to chaotic. Corresponding, the 

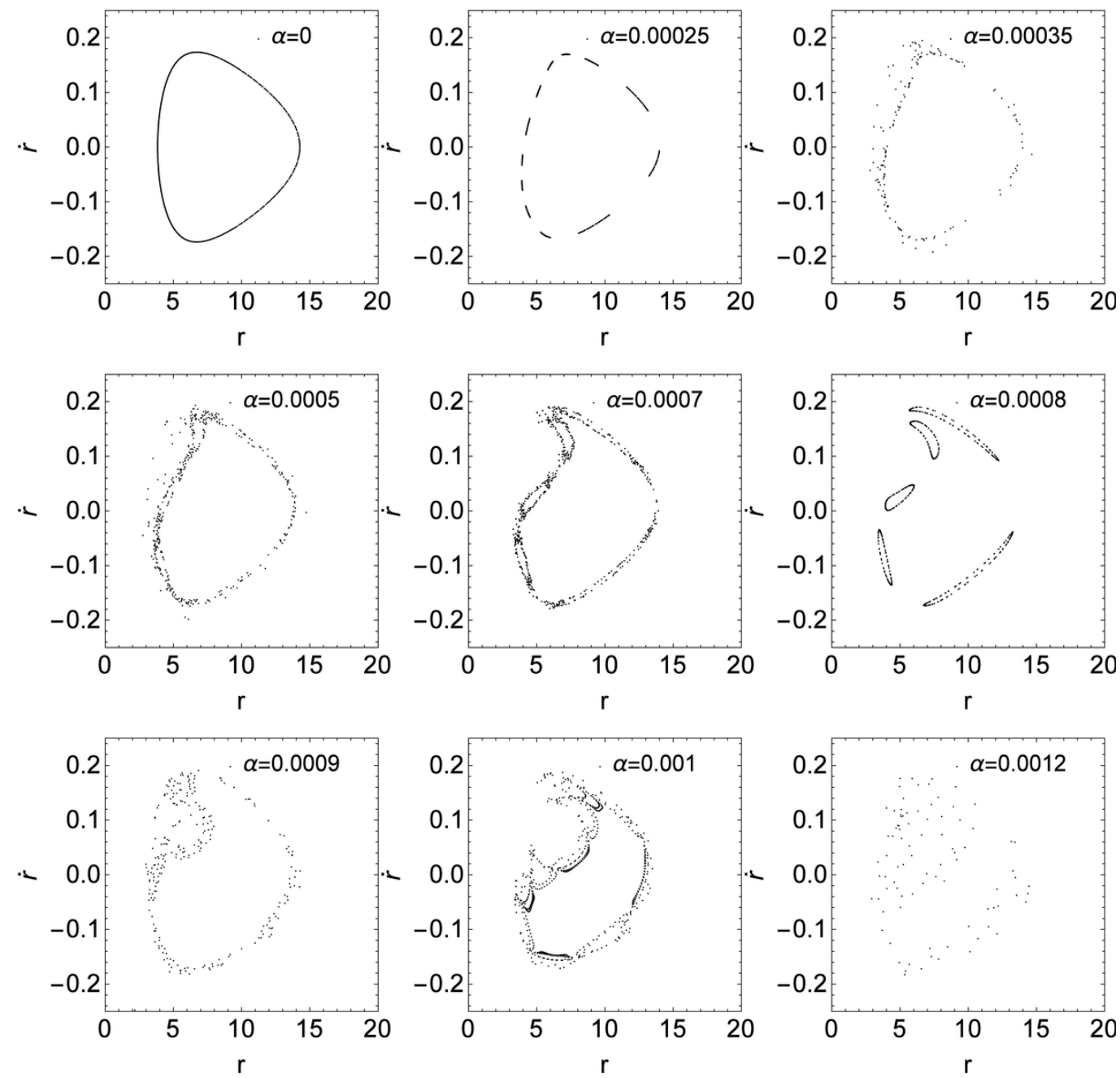

Figure 8. The change of Poincaré section $\left(\theta=\frac{\pi}{2}\right.$ on the plane $\left.(r, \dot{r})\right)$ with acceleration parameter $\alpha$ for the motion of the timelike particle in the accelerating and rotating black hole spacetime with the fixed parameters $a=0.6, E=0.95, L=3 M$ and the initial conditions $\{r(0)=12 ; \dot{r}(0)=0.1$; $\left.\theta(0)=\frac{\pi}{2}\right\}$.

non-integrability of the motion of particle in figure 8 first increases and then decreases, and finally increases with $\alpha$. Similarly, for the larger $\alpha$, we obtain only a kind of unstable escaped solutions for this chosen initial condition as in the previous discussion. Thus, the dependence of the non-integrability of the motion on the acceleration parameter $\alpha$ depends on the initial conditions and the parameters of system.

In figures 9 and 10, we also plot Poincaré section on the plane $(r, \dot{r})$ for the motion of the timelike particle with different initial conditions in the background of accelerating and rotating black hole spacetime with different parameters. According to previous discussion, we here set the acceleration parameter in the range $0 \leq \alpha<0.0016$. From figure 9, we find that for the fixed rotation parameter $a=0.6$, the chaotic region first increases and then decreases with the increase of the acceleration parameter $\alpha$. Moreover, for the case 

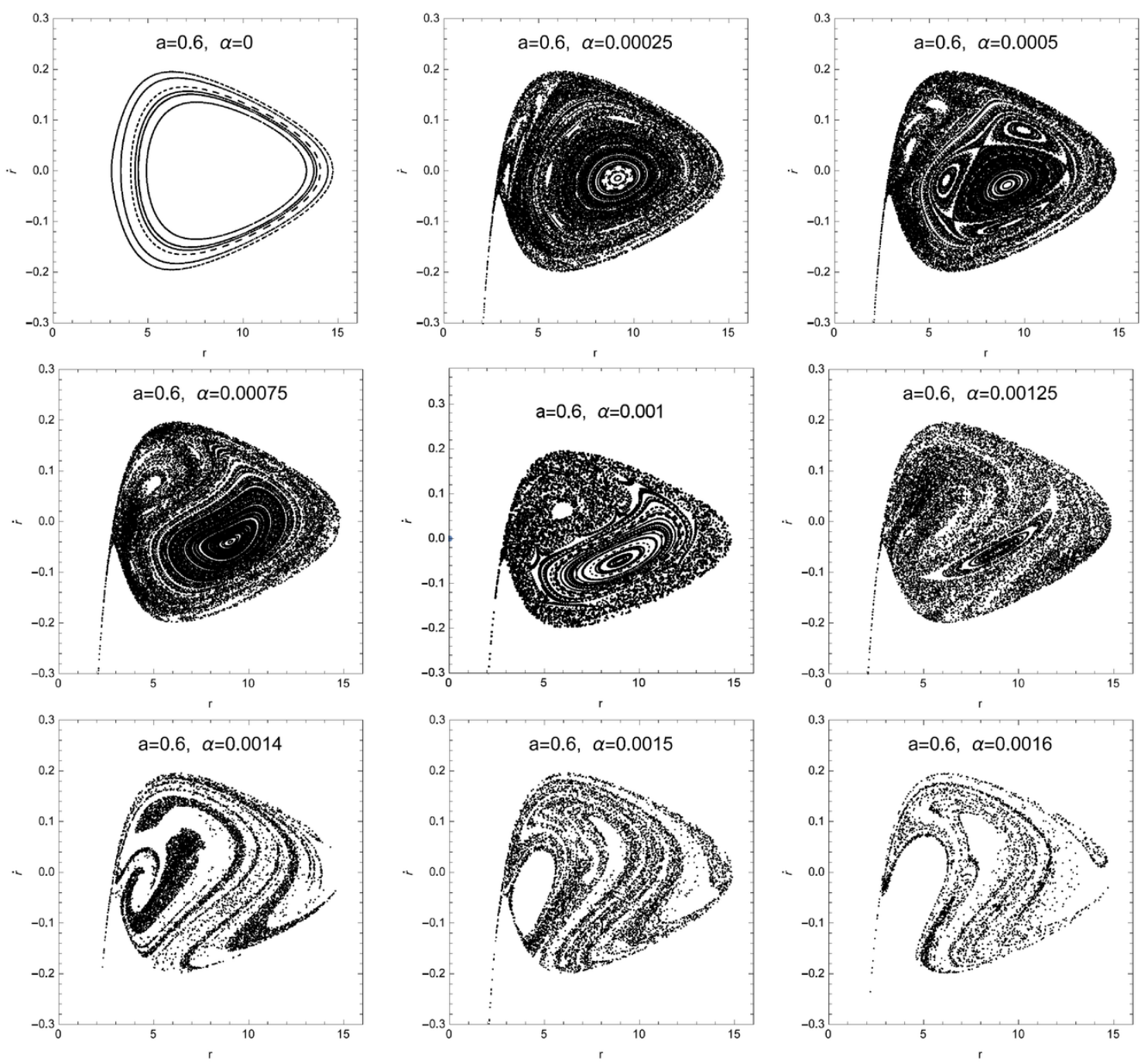

Figure 9. The change of Poincaré section $\left(\theta=\frac{\pi}{2}\right.$ on the plane $\left.(r, \dot{r})\right)$ with the acceleration parameter $\alpha$ for the motion of the timelike particle in the accelerating and rotating black hole spacetime with the fixed parameters $a=0.6, E=0.95$ and $L=3 M$.

$\alpha=0$, we find that there are a series of close curves in the Poincaré section, which means that there do not exist chaotic orbits in the Kerr black hole spacetime, which is consistent with the previous discussion. Figure 10 tells us that for the fixed acceleration parameter $\alpha=0.001$, the chaotic region first increases and then decreases with the increase of the rotation parameter $a$. It is shown clearly in the Poincaré section that the numbers and positions of fixed points of the system change with the rotation parameter $a$.

The dependence of dynamical behaviors of system on the black hole parameters can also be visualised in the form of a bifurcation diagram. In figures 11 and 12, we plot the bifurcation diagram of the radial coordinate $r$ with the acceleration parameter $\alpha$ and the rotation parameter $a$ for the particle motion in the accelerating and rotating black hole spacetime with fixed $E=0.95$ and $L=3 M$. Here we chose the set of initial conditions 

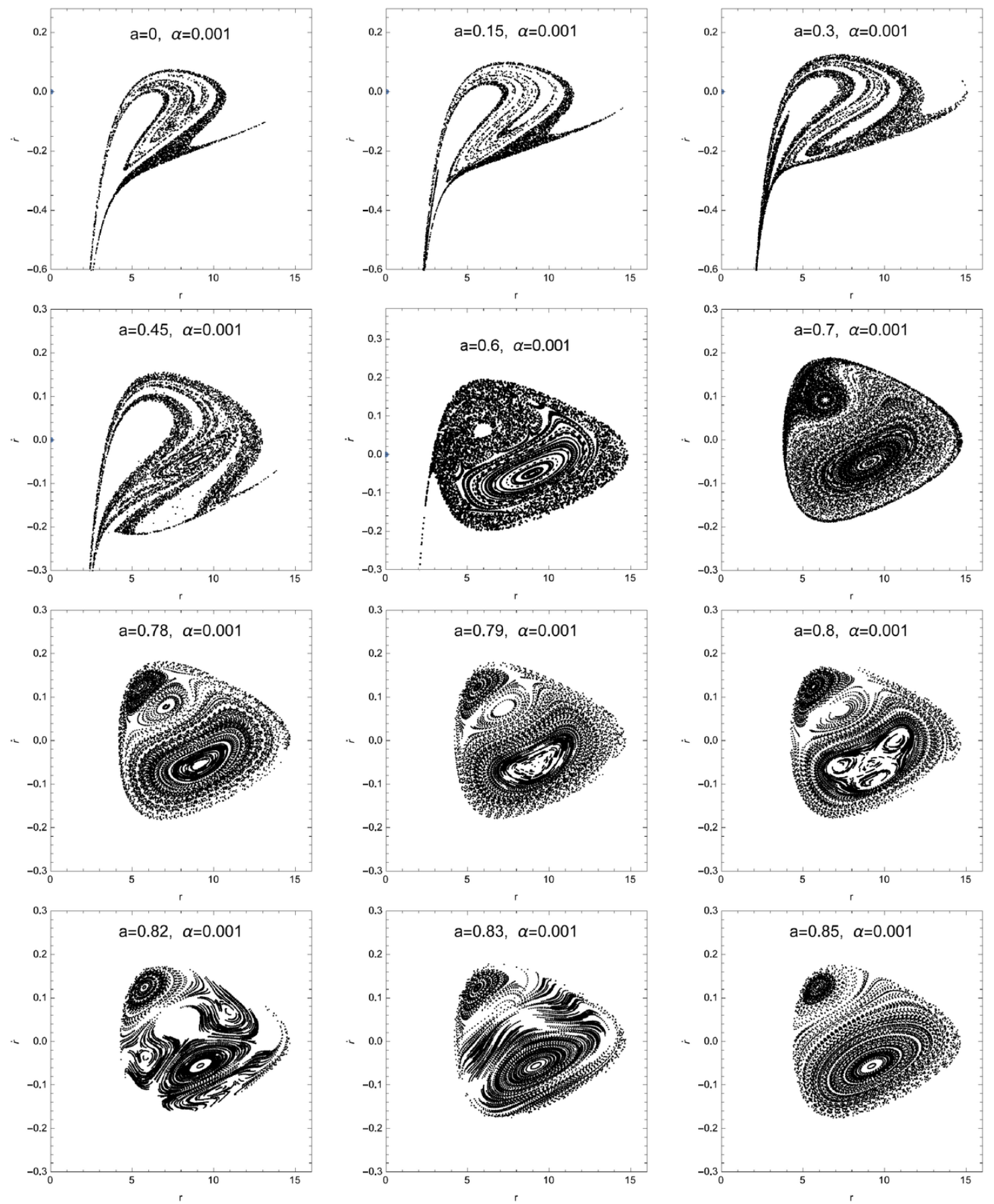

Figure 10. The change of Poincaré section $\left(\theta=\frac{\pi}{2}\right.$ on the plane $\left.(r, \dot{r})\right)$ with the rotation parameter $a$ for the motion of the timelike particle in the accelerating and rotating black hole spacetime with the fixed parameters $\alpha=0.001, E=0.95$ and $L=3 M$.

are $\left\{r(0)=8 ; \dot{r}(0)=0 ; \theta(0)=\frac{\pi}{2}\right\}$. We find that for the case $\alpha=0$ there is only a periodic solution and no bifurcation for the dynamical system (2.13), which confirms again that the motions of particles are not chaotic in the rotation black hole spacetime 

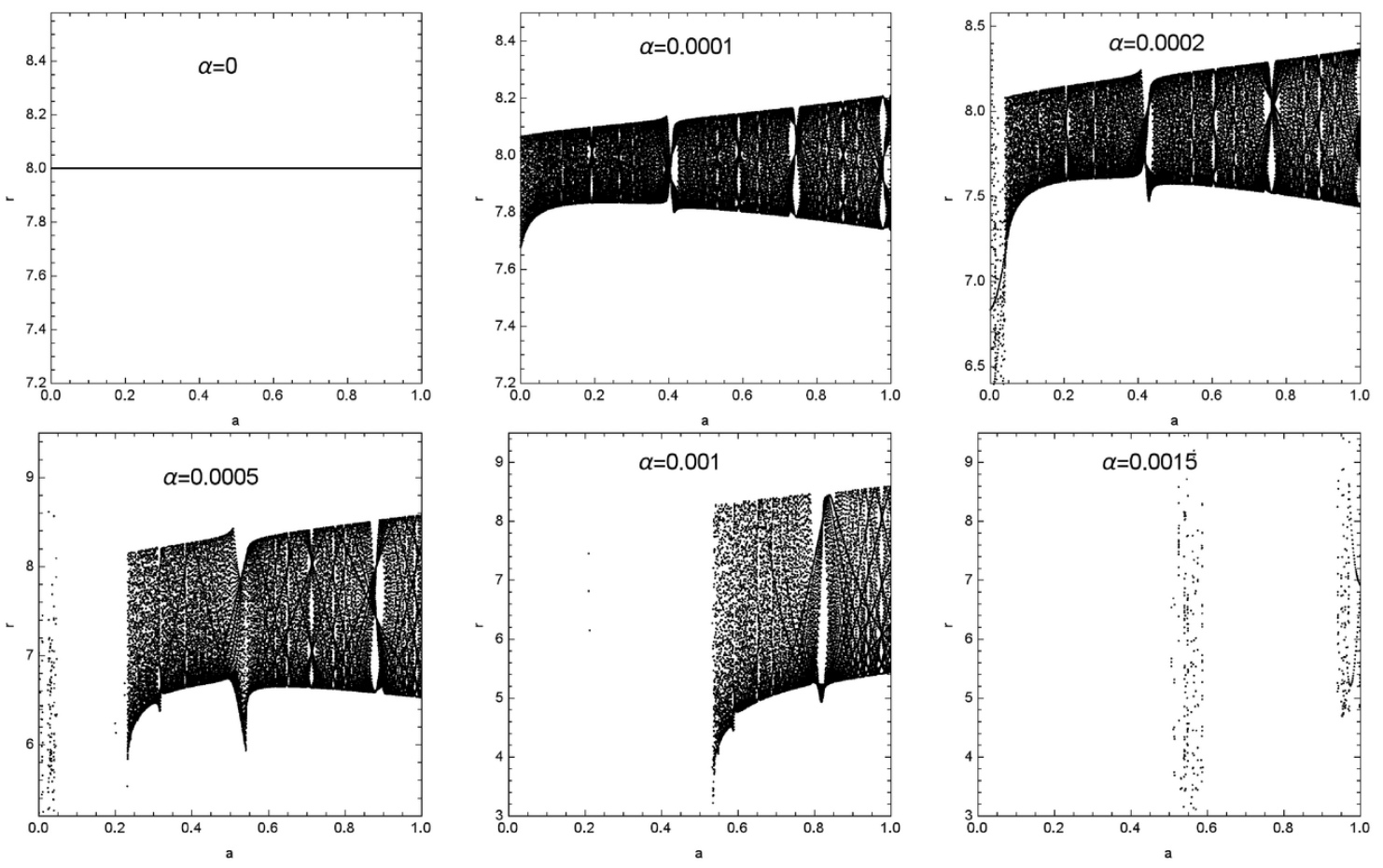

Figure 11. The bifurcation with the rotation parameter $a$ for the motion of the timelike particle with the parameters $E=0.95$ and $L=3 M$ in the accelerating and rotating black hole spacetime. The set of initial conditions are $\left\{r(0)=8 ; \dot{r}(0)=0 ; \theta(0)=\frac{\pi}{2}\right\}$.

without acceleration. For the case with non-zero acceleration, it is obvious that there exist periodic, chaotic and escaped solutions which depend on the acceleration parameter $\alpha$ and the rotation parameter $a$. For the chaotic solution, one can find the range of $r$ in the bifurcation diagram increases almost with the acceleration parameter $\alpha$, which means that $\alpha$ enhances the strength of chaotic motion. With increase of the rotation parameter $a$, the range of $r$ in the chaos solution first decreases and then increases for the smaller $\alpha$, but it decreases for the larger $\alpha$. Moreover, we find that the range of $a$ in which there exists escaped solution increases with $\alpha$. This could be explained by a fact that for the larger $\alpha$ the acceleration horizon $r_{A}$ is closer to the event horizon $r_{H}$ so that the particle is out-off-balance which yields that the particle falls either into the event horizon or into the acceleration horizon of the black hole in this case. These results show that the acceleration brings richer properties for the geodesic motion of particles in the accelerating and rotating black hole spacetime.

\section{Summary}

In summary, we have studied the motion of timelike particles along geodesic in the background of accelerating and rotating black hole spacetime by Poincaré sections, the frequency spectrum and the power spectrum, the fast Lyapunov exponent indicator, and the bifurcation diagram. Our results confirm that the chaos exists in the geodesic motion of the 

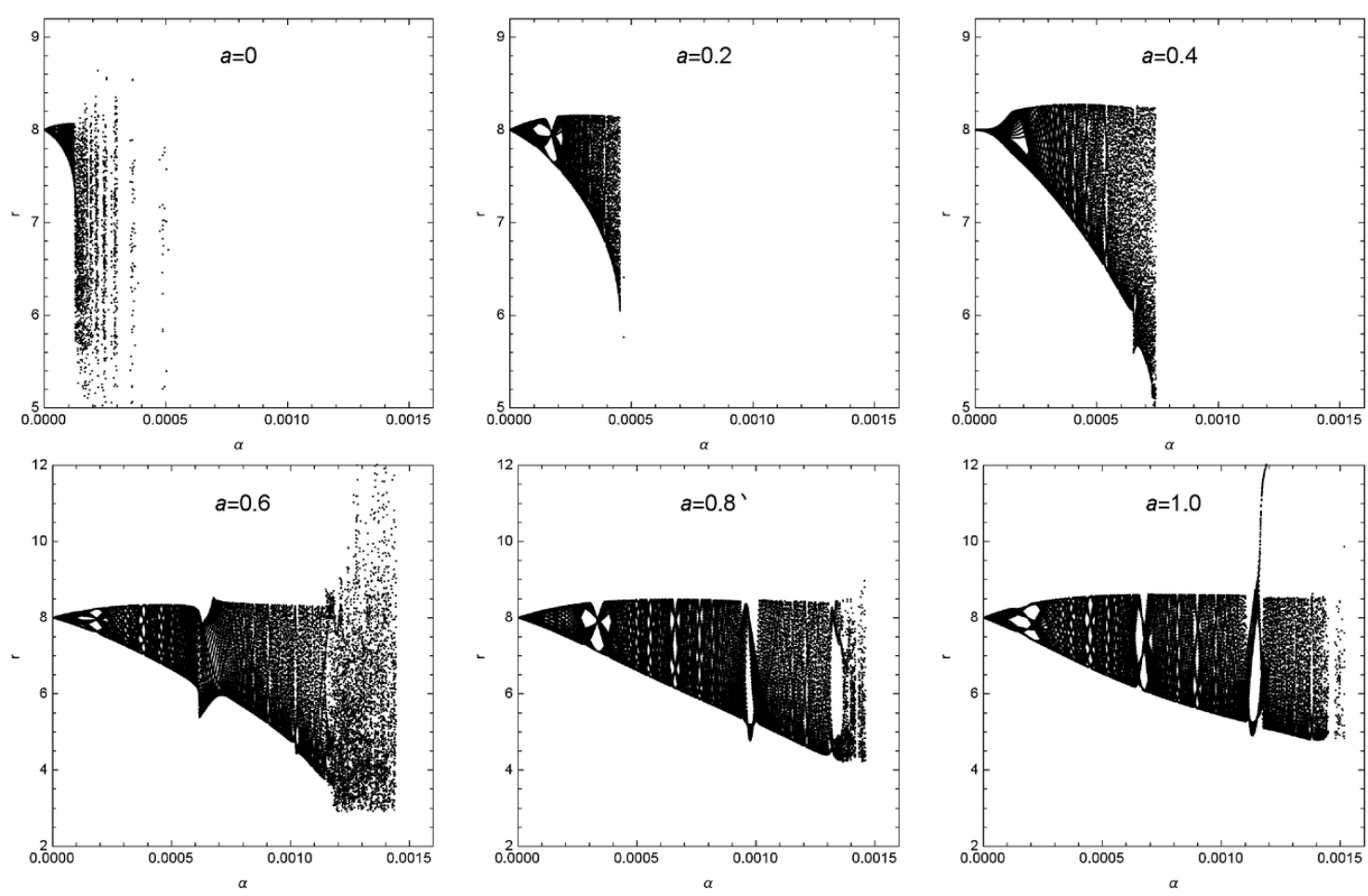

Figure 12. The bifurcation with the acceleration parameter $\alpha$ for the motion of the timelike particle with the parameters $E=0.95$ and $L=3 M$ in the accelerating and rotating black hole spacetime. The set of initial conditions are $\left\{r(0)=8 ; \dot{r}(0)=0 ; \theta(0)=\frac{\pi}{2}\right\}$.

particles. It is mainly because the presence of the acceleration parameter $\alpha$ yields that the equations of motion are not be variable-separable and the corresponding dynamical system is non-integrable. Moreover, we probe the effects of the acceleration and rotation parameters of black hole on the chaotic behavior of a timelike geodesic particle. Our results show that the dependence of the non-integrability and the chaotic motion on the acceleration parameter $\alpha$ depends on the initial conditions and the parameters of system. For the fixed acceleration parameter $\alpha=0.001$, we find that the chaotic region in Poincaré sections first increases and then decreases with the increase of the rotation parameter $a$. For the fixed rotation parameter $a=0.6$, the chaotic region first increases and then decreases with $\alpha$. For the particle with chaotic motion, we find the range of $r$ in the bifurcation diagram increases almost with the acceleration parameter $\alpha$, which means that $\alpha$ enhances the strength of chaotic motion. With increase of the rotation parameter $a$, the range of $r$ in the chaos solution first decreases and then increases for the smaller $\alpha$, but it decreases for the larger $\alpha$. When $\alpha=0$, we find that it can be reduced to the case of Kerr black hole spacetime in which there does not exists chaotic motion of particle. Our results show that the acceleration yields richer effects on the geodesic motion of particles in the accelerating and rotating black hole spacetime. 


\section{Acknowledgments}

We would like to thank Prof. Wenhua Hai and Prof. Guishu Chong for their useful discussions of the early stage of this work. This work was partially supported by the National Natural Science Foundation of China under Grant No. 11275065, No. 11475061, the construct program of the National Key Discipline, and the Open Project Program of State Key Laboratory of Theoretical Physics, Institute of Theoretical Physics, Chinese Academy of Sciences, China (No. Y5KF161CJ1).

Open Access. This article is distributed under the terms of the Creative Commons Attribution License (CC-BY 4.0), which permits any use, distribution and reproduction in any medium, provided the original author(s) and source are credited.

\section{References}

[1] J.C. Sprott, Chaos and time-series analysis, Oxford University Press, Oxford U.K. (2003).

[2] E. Ott, Chaos in dynamical systems, second edition, Cambridge University Press, Cambridge U.K. (2002).

[3] R. Brown and L.O. Chua, Clarifying chaos: examples and counterexamples, Int. J. Bifurcat. Chaos 6 (1996) 219.

[4] R. Brown and L.O. Chua, Clarifying chaos II: Bernoulli chaos, zero Lyapunov exponents and strange attractors, Int. J. Bifurcat. Chaos 8 (1998) 1.

[5] B. Carter, Global structure of the Kerr family of gravitational fields, Phys. Rev. 174 (1968) 1559 [INSPIRE].

[6] C.P. Dettmann, N.E. Frankel and N.J. Cornish, Fractal basins and chaotic trajectories in multi-black-hole spacetimes, Phys. Rev. D 50 (1994) R618 [gr-qc/9402027] [INSPIRE].

[7] W. Hanan and E. Radu, Chaotic motion in multi-black hole spacetimes and holographic screens, Mod. Phys. Lett. A 22 (2007) 399 [gr-qc/0610119] [INSPIRE].

[8] S.D. Majumdar, A class of exact solutions of Einstein's field equations, Phys. Rev. 72 (1947) 390 [INSPIRE].

[9] A. Papaetrou, A static solution of the equations of the gravitational field for an arbitrary charge distribution, Proc. Roy. Irish Acad. A 51 (1947) 191 [INSPIRE].

[10] L. Bombelli and E. Calzetta, Chaos around a black hole, Class. Quant. Grav. 9 (1992) 2573 [INSPIRE].

[11] J.M. Aguirregabiria, Chaotic scattering around black holes, Phys. Lett. A 224 (1997) 234 [gr-qc/9604032] [INSPIRE].

[12] Y. Sota, S. Suzuki and K.-i. Maeda, Chaos in static axisymmetric spacetimes: 1. Vacuum case, Class. Quant. Grav. 13 (1996) 1241 [gr-qc/9505036] [INSPIRE].

[13] V. Witzany, O. Semerák and P. Suková, Free motion around black holes with discs or rings: between integrability and chaos - IV, Mon. Not. Roy. Astron. Soc. 451 (2015) 1770 [arXiv: 1503.09077] [INSPIRE].

[14] V. Karas and D. Vokrouhlický, Chaotic motion of test particles in the Ernst space-time, Gen. Rel. Grav. 24 (1992) 729. 
[15] J.R. Gair, C. Li and I. Mandel, Observable properties of orbits in exact bumpy spacetimes, Phys. Rev. D 77 (2008) 024035 [arXiv: 0708.0628] [inSPIRE].

[16] G. Contopoulos, G. Lukes-Gerakopoulos and T.A. Apostolatos, Orbits in a non-Kerr dynamical system, Int. J. Bifurcat. Chaos 21 (2011) 2261 [arXiv:1108.5057] [INSPIRE].

[17] G. Lukes-Gerakopoulos, G. Contopoulos and T.A. Apostolatos, Non-linear effects in non-Kerr spacetimes, Springer Proc. Phys. 157 (2014) 129 [arXiv: 1408.4697] [InSPIRE].

[18] F.L. Dubeibe, L.A. Pachon and J.D. Sanabria-Gomez, Chaotic dynamics around astrophysical objects with nonisotropic stresses, Phys. Rev. D 75 (2007) 023008 [gr-qc/0701065] [INSPIRE].

[19] E. Gueron and P.S. Letelier, Geodesic chaos around quadrupolar deformed centers of attraction, Phys. Rev. E 66 (2002) 046611 [INSPIRE].

[20] H. Varvoglis and D. Papadopoulos, Chaotic interaction of charged particles with a gravitational wave, Astron. Astrophys. 261 (1992) 664.

[21] A.V. Frolov and A.L. Larsen, Chaotic scattering and capture of strings by black hole, Class. Quant. Grav. 16 (1999) 3717 [gr-qc/9908039] [INSPIRE].

[22] L.A. Pando Zayas and C.A. Terrero-Escalante, Chaos in the gauge/gravity correspondence, JHEP 09 (2010) 094 [arXiv: 1007.0277] [INSPIRE].

[23] D.-Z. Ma, J.-P. Wu and J. Zhang, Chaos from the ring string in a Gauss-Bonnet black hole in $A d S_{5}$ space, Phys. Rev. D 89 (2014) 086011 [arXiv:1405.3563] [InSPIRE].

[24] I. Bengtsson and P. Sandin, Anti de Sitter space, squashed and stretched, Class. Quant. Grav. 23 (2006) 971 [gr-qc/0509076] [InSPIRE].

[25] J.B. Griffiths and J. Podolský, Accelerating and rotating black holes, Class. Quant. Grav. 22 (2005) 3467 [gr-qc/0507021] [INSPIRE].

[26] J. Podolský and J.B. Griffiths, Accelerating Kerr-Newman black holes in (anti-)de Sitter space-time, Phys. Rev. D 73 (2006) 044018 [gr-qc/0601130] [INSPIRE].

[27] M. Bilal and K. Saifullah, Thermodynamics of accelerating and rotating black holes, Astrophys. Space Sci. 343 (2013) 165 [arXiv:1010.5575] [INSPIRE].

[28] U.A. Gillani, M. Rehman and K. Saifullah, Hawking radiation of scalar particles from accelerating and rotating black holes, JCAP 06 (2011) 016 [arXiv:1102.0029] [INSPIRE].

[29] W.-P. Yao, S. Chen, C. Liu and J. Jing, Effects of acceleration on the collision of particles in the rotating black hole spacetime, Eur. Phys. J. C 72 (2012) 1898 [arXiv:1105.6156] [INSPIRE].

[30] S.W. Hawking and S.F. Ross, Pair production of black holes on cosmic strings, Phys. Rev. Lett. 75 (1995) 3382 [gr-qc/9506020] [INSPIRE].

[31] J. Podolský and H. Kadlecová, Radiation generated by accelerating and rotating charged black holes in (anti-)de Sitter space, Class. Quant. Grav. 26 (2009) 105007 [arXiv: 0903.3577] [INSPIRE].

[32] J.F. Plebański and M. Demiański, Rotating, charged and uniformly accelerating mass in general relativity, Annals Phys. 98 (1976) 98 [INSPIRE].

[33] X. Wu, T.Y. Huang, X.S. Wan and H. Zhang, Comparison among correction methods of individual Kepler energies in n-body simulations, Astron. J. 133 (2007) 2643. 
[34] D.Z. Ma, X. Wu and J.F. Zhu, Velocity scaling method to correct individual Kepler energies, New Astron. 13 (2008) 216.

[35] D.Z. Ma, X. Wu and F.Y. Liu, Velocity corrections to Kepler energy and Laplace integral, Int. J. Mod. Phys. C 19 (2008) 1411.

[36] D.Z. Ma, X. Wu and S.Y. Zhong, Extending Nacozy's approach to correct all orbital elements for each of multiple bodies, Astrophys. J. 687 (2008) 1294.

[37] V.I. Osedelec, A multiplicative ergodic theorem. Lyapunov characteristic numbers for dynamical systems, Trans. Moscow Math. Soc. 19 (1968) 197.

[38] A.E. Motter, Relativistic chaos is coordinate invariant, Phys. Rev. Lett. 91 (2003) 231101 [gr-qc/0305020] [INSPIRE].

[39] J.C. Sprott, http://sprott.physics.wisc.edu/chaos/lyapexp.htm.

[40] C. Froeschlé, E. Lega and R. Gonczi, Fast Lyapunov indicators. Application to asteroidal motion, Celest. Mech. Dyn. Astron. 67 (1997) 41.

[41] C. Froeschlé and E. Lega, On the structure of symplectic mappings. The fast Lyapunov indicator: a very sensitive tool, Celest. Mech. Dyn. Astron. 78 (2000) 167.

[42] M. Fouchard, E. Lega, C. Froeschlé and C. Froeschlé, On the relationship between fast Lyapunov indicator and periodic orbits for continuous flows, Celest. Mech. Dyn. Astron. 83 (2002) 205.

[43] G. Benettin, L. Galgani and J.M. Strelcyn, Kolmogorov entropy and numerical experiments, Phys. Rev. A 14 (1976) 2338.

[44] G. Tancredi, A. Sánchez and F. Roig, A comparison between methods to compute Lyapunov exponents, Astron. J. 121 (2001) 1171.

[45] X. Wu and T.-y. Huang, Computation of Lyapunov exponents in general relativity, Phys. Lett. A 313 (2003) 77 [gr-qc/0302118] [INSPIRE].

[46] X. Wu and $\mathrm{H}$. Zhang, Chaotic dynamics in a superposed Weyl spacetime, Astrophys. J. 652 (2006) 1466.

[47] X. Wu, T.Y. Huang and H. Zhang, Lyapunov indices with two nearby trajectories in a curved spacetime, Phys. Rev. D 74 (2006) 083001 [arXiv:1006.5251] [INSPIRE]. 